\title{
Sliding Mode Observer and Event Triggering Mechanism Co-design
}

\author{
Houssem Eddine Amara \\ Electrical Engineering Department \\ University Ferhat Abbas Setif 1 \\ Setif, Algeria \\ houssem_am@hotmail.fr \\ Mohamed Amine Sid \\ Electrical Engineering Department \\ University Ferhat Abbas Setif 1 \\ Setif, Algeria \\ amineatweb@yahoo.fr
}

\author{
Samia Latreche \\ Electrical Engineering Department \\ University Ferhat Abbas Setif 1 \\ Setif, Algeria \\ ksamia2002@yahoo.fr \\ Mabrouk Khemliche \\ Electrical Engineering Department \\ University Ferhat Abbas Setif 1 \\ Setif, Algeria \\ mabroukkhemliche@univ-setif.dz
}

\begin{abstract}
Sliding mode observer and event triggering mechanism co-design is the subject of this paper. The synthesis of a sliding mode observer under any event triggering mechanism is given, where the proposed observer can be viewed as a special structure of the Kalman filter. Then, the event triggering condition is updated according to the coverage index value computed with the help of the reachability set representation. A numerical example illustrates the effectiveness of the proposed approach.
\end{abstract}

Keywords-sliding mode observer; event-based scheduling; codesign; estimation performance; communication design optimization

\section{INTRODUCTION}

Many estimator design methods have been developed under the assumption that the measurement vector is periodically available. However, sensors may not feed-back the estimator periodically, due to several reasons such as limited network bandwidth and high energy consumption of the radio unit for wireless sensors. In this case, the classic periodic sampling can be replaced by an event-based technique, which insures acceptable estimation performance with limited network resources. Achieving a desired balance between network state estimator quality and sensor to estimator communication rate has led to the use of robust observer methods and in this context sliding mode observer is a very popular tool. The sliding mode observers are proposed with the idea to drive the dynamics of a system to an sliding manifold, that is an integral manifold with finite reaching time [1]. The first sliding mode has been discussed in [2-4]. In addition, some SMO have attractive properties similar to those of the Kalman filter (i.e. noise resilience) [5], but with a simpler implementation [6]. Sometimes this design can be performed by applying an equivalent control method $[7,8]$, allowing the proposal of robust to noise observers, since the equivalent control is slightly affected by noisy measurements. A key feature in the Utkin observer [9] is the introduction of a switching function in the observer to achieve a sliding mode and also stable error dynamics. For better utilization of shared communication and processing resources or reduction of hardware costs we deal with event-based designs. The area of event-based control and estimation has substantially grown during the last decades [1012]. In [13,] an event-triggered approach was used to trigger the data transmission from a sensor to a remote observer. In [14], a sensor data scheduling problem was considered and a feedback policy to choose the transmission times which provides a trade-off between the communication rate and the estimation error was used. The objective in [15] was the development of predictive triggering mechanisms for eventbased state estimation.

The main contribution of this paper is the introduction of an event-based technique and a communication design optimization with a corresponding estimation by adopting a sliding mode from which we obtain high estimation quality with low energy consumption by minimizing the number of sensors.

\section{PROBLEM FORMULATION}

The event-based sliding mode state estimation process is illustrated in Figure 1.

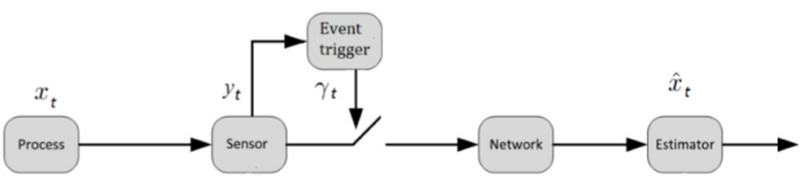

Fig. 1. Event-based sliding mode state estimation (1): 


$$
\left\{\begin{array}{l}
\dot{x}_{t}=A x_{t}+B u_{t} \\
y_{t}=C x_{t}
\end{array}\right.
$$

where: $\mathrm{x} \in \mathfrak{K}^{\mathrm{n}_{\mathrm{x}}}, \mathcal{Y} \in \mathfrak{K}^{\mathrm{n}_{\mathrm{y}}}, \mathrm{u} \in \mathfrak{K}^{\mathrm{n}_{\mathrm{u}}}$, and the matrices A, B, C have the appropriate dimensions. Without loss of generality, we assume that $\mathrm{C} \in \mathcal{R}^{\mathrm{n}_{\mathrm{y}} \times \mathrm{n}_{\mathrm{x}}}$ has full row rank which means each of the measured outputs is independent.

Let $\varepsilon_{\mathrm{t}} \triangleq \mathcal{Y}_{\mathrm{t}}-\mathcal{Y}_{\mathcal{T}_{\mathrm{t}}}$, where $\mathcal{T}_{\mathrm{t}}$ denotes the previous time instant when the measurement of sensor is transmitted defined as follows:

$$
\mathcal{T}_{\tau}=\left\{\begin{array}{c}
\mathcal{T}_{t}^{-} \text {if } \gamma_{t}=0 \\
\text { t if } \gamma_{t}=1
\end{array}, \text { and } \mathcal{T}_{0}^{-}=0\right.
$$

where $\mathcal{T}_{\mathfrak{t}}^{-}$denotes the left hand limit of $\mathcal{T}_{\tau}$ at the instant $t$. The main advantage of using the send-on-delta conditions is that feedback communication from the estimator is not needed as the conditions only depend on the sensor measurements. This fact reduces hardware and energy consumption cost and facilitates the implantation of event-triggering schemes. The objective is to obtain an estimate of the state $\mathrm{x}_{\mathrm{t}}$ based only on the knowledge of the quantities $\mathrm{y}_{\mathcal{J}_{\mathrm{t}}}$ and $\mathrm{u}_{\mathrm{t}}$. In the simplest form of the sliding mode observer, instead of feeding back the output error between the observer and the system in a linear fashion, the output error is fed back via a discontinuous switched signal. Furthermore, we consider the following EB sensor data scheduler:

$$
\gamma_{\mathrm{t}}=\left\{\begin{array}{cc}
0, & \text { if }\left\|\epsilon_{\mathrm{t}}\right\|_{\infty} \leq \zeta, \\
1, & \text { otherwise }
\end{array}\right.
$$

where $\zeta \leq \infty$ is a predefined threshold and $\epsilon_{\mathrm{t}}$ the output error.

The objective of this article is to find or approximate an optimal state estimate of EB sensor data scheduler (3), which we call nonlinear EB state estimation or EB state estimation problem interchangeably. In the next section, a sliding mode framework of the EB state estimation problem is presented.

\section{EVENT BASED SLIDING MODE OBSERVER}

\section{A. Similarity Transformation}

Consider the transformation $\mathrm{x}=\mathrm{T}_{\mathrm{c}}^{-1} \mathrm{x}_{1}$ associated with the invertible matrix:

$$
\mathrm{T}_{\mathrm{c}}=\left[\begin{array}{c}
\mathrm{N}_{\mathrm{C}}^{\mathrm{T}} \\
\mathrm{C}
\end{array}\right]
$$

where $\mathrm{N}_{\mathrm{c}} \in \mathcal{R}^{\mathrm{n}_{\mathrm{x}} \times\left(\mathrm{n}_{\mathrm{x}}-\mathrm{n}_{\mathrm{y}}\right)}$ is a full rank column matrix composed with the null space basis of $\mathrm{C}$. By substitution we obtain:

$$
\left\{\begin{array}{c}
\dot{\mathrm{x}}_{1}(\mathrm{t})=\breve{\mathrm{A}} \mathrm{x}_{1}(\mathrm{t})+\breve{\mathrm{B}} \mathrm{u}(\mathrm{t}) \\
y(\mathrm{t})=\check{C} \mathrm{x}_{1}(\mathrm{t})
\end{array}\right.
$$

where:

$$
\check{\mathrm{A}}=\mathrm{T}_{\mathrm{c}} \mathrm{AT}_{\mathrm{c}}^{-1}, \breve{\mathrm{B}}=\mathrm{T}_{\mathrm{c}} \mathrm{B} \text {, and } \check{\mathrm{C}}=\mathrm{CT}_{\mathrm{c}}^{-1}=\left[\begin{array}{ll}
0 & \mathrm{I}_{\mathrm{p}}
\end{array}\right](6)
$$

An observer for (1) was proposed as follows [9]:

$$
\left\{\begin{array}{c}
\dot{\hat{x}}_{\mathrm{t}}=A \hat{x}_{\mathrm{t}}+B \mathrm{Bu}_{\mathrm{t}}+\mathrm{G}_{\mathrm{n}} \mathrm{v} \\
\hat{\mathrm{y}} \mathrm{t}=\mathrm{C} \hat{\mathrm{x}}_{\mathrm{t}}
\end{array}\right.
$$

where $(\hat{\mathrm{x}}, \hat{\mathcal{Y}})$ are the estimates of $\mathrm{x}, \mathcal{Y}$ and $\mathrm{v}$ is a discontinuous injection term. $e_{t}=\hat{x}_{t}-x_{t}$ and $\check{e}_{y}(t)=\hat{y} t-\mathcal{Y}_{\mathcal{T}_{t}}$ are defined as the state estimation and output estimation errors respectively. The term $\mathrm{v}$ is defined component-wise as:

$$
\mathrm{v}_{\mathrm{i}}=\rho \operatorname{sign}\left(\tilde{\mathrm{e}}_{y, \mathrm{i}}\right), \mathrm{i}=1, \ldots, \mathrm{p}
$$

where $\rho$ is a positive scalar and $\tilde{\mathrm{e}}_{\mathrm{y}, \mathrm{i}}$ represents the $\mathrm{i}^{\text {th }}$ component of $\tilde{\mathrm{e}}_{y}$. The term $\mathrm{v}$ is designed to be discontinuous with respect to the sliding surface $S=\{e: C e=0\}$ to force the trajectories of $\mathrm{e}(\mathrm{t})$ into $\mathrm{S}$ in finite time. Assuming, without loss of generality, that the system is already in the coordinate associated with (6), then the gain $G_{n}$ has the following structure:

$$
\mathrm{G}_{\mathrm{n}}=\left[\begin{array}{c}
\mathrm{L} \\
-\mathrm{I}_{\mathrm{p}}
\end{array}\right]
$$

where $\mathrm{L} \in \mathcal{R}^{(\mathrm{n}-\mathrm{p}) \times \mathrm{p}}$ represents the design freedom, following the definition of $\mathrm{e}(\mathrm{t})$ and (1). The error system is given by:

$$
\mathrm{e}(\mathrm{t})=\mathrm{Ae}(\mathrm{t})+\mathrm{G}_{\mathrm{n}} \mathrm{V}
$$

From the structure of the output distribution matrix $\mathrm{C}$ in (6) the state estimation error can be partitioned as $\mathrm{e}=\mathrm{col}\left(\mathrm{e}_{1}, \mathrm{e}_{y}\right)$, where $\mathrm{e}_{1} \in \mathcal{R}^{\mathrm{n}-\mathrm{p}}$. Consequently the error system from (10) can be written in the form:

$$
\begin{aligned}
& \mathrm{e}_{1}(\mathrm{t})=\mathrm{A}_{21} \mathrm{e}_{1}(\mathrm{t})+\mathrm{A}_{12} \mathrm{e}_{y}(\mathrm{t})+\mathrm{Lv} \\
& \mathrm{e}_{y}(\mathrm{t})=\mathrm{A}_{21} \mathrm{e}_{1}(\mathrm{t})+\mathrm{A}_{12} \mathrm{e}_{y}(\mathrm{t})-\mathrm{v}
\end{aligned}
$$

Furthermore, (12) can be written component-wise as:

$$
\dot{e}_{y, i}(t)=A_{21, i} e_{1}(t)+A_{22, i} e_{y}(t)-\operatorname{psign}\left(\tilde{e}_{y, i}\right)
$$

where $A_{21, i}$ and $A_{22, i}$ represent the $i^{\text {th }}$ rows of $A_{21}$ and $A_{22}$ respectively. To develop the conditions under which the sliding will take place, the reachability condition will be tested.

Notice that $\mathrm{e}_{y, \mathrm{i}}=\mathcal{y}_{\mathrm{i}}-\tilde{y}_{\mathrm{i}}$ and $\tilde{\mathrm{e}}_{y_{, \mathrm{i}}}=\mathcal{y}_{\mathrm{t}, \mathrm{i}}-\tilde{y}_{\mathrm{i}}$. Then $\mathrm{e}_{y, \mathrm{i}}-\tilde{\mathrm{e}}_{y, \mathrm{i}}=\mathcal{Y}_{\mathrm{i}}-\mathcal{Y}_{\mathrm{t}, \mathrm{i}}$ and from (3) we get:

$$
\left|\mathrm{e}_{y, \mathrm{i}}\right|-\zeta \leq\left|\tilde{\mathrm{e}}_{\mathcal{y}, \mathrm{i}}\right|
$$

From (13) and (14) we obtain:

$$
\begin{aligned}
& \mathrm{e}_{y, \mathrm{i}} \dot{\mathrm{e}}_{y, \mathrm{i}}=\mathrm{e}_{y, \mathrm{i}}\left(\mathrm{A}_{21, \mathrm{i}} \mathrm{e}_{1}+\mathrm{A}_{22, \mathrm{i}} \mathrm{e}_{y}\right)-\rho\left|\left(\tilde{\mathrm{e}}_{y, \mathrm{i}}\right)\right| \\
& \leq \mathrm{e}_{y, \mathrm{i}}\left(\mathrm{A}_{21, \mathrm{i}} \mathrm{e}_{1}+\mathrm{A}_{22, \mathrm{i}} \mathrm{e}_{y}\right)-\rho\left|\left(\mathrm{e}_{y, \mathrm{i}}\right)\right|+\rho \zeta \\
& <-\left|\left(\mathrm{e}_{y, \mathrm{i}}\right)\right|\left(\rho-\left|\left(\mathrm{A}_{21, \mathrm{i}} \mathrm{e}_{1}+\mathrm{A}_{22, \mathrm{i}} \mathrm{e}_{y}\right)\right|-\frac{\rho \zeta}{\left|\left(\mathrm{e}_{y, \mathrm{i}}\right)\right|}\right.
\end{aligned}
$$

Provided that the scalar $\mathrm{p}$ is chosen such (large enough) that:

$$
\mathrm{p}>\left|\left(\mathrm{A}_{21, \mathrm{i}} \mathrm{e}_{1}+\mathrm{A}_{22, \mathrm{i}} \mathrm{e}_{\mathrm{y}}\right)\right|-\frac{\rho \zeta}{\left|\left(\mathrm{e}_{y, \mathrm{i}}\right)\right|}+\mathrm{n}
$$

where the scalar $\mathrm{n} \in \mathfrak{R}_{+}$. Then :

$$
\mathrm{e}_{y, i} \dot{\mathrm{e}}_{y, \mathrm{i}}<-\mathrm{n}\left|\mathrm{e}_{y, \mathrm{i}}\right|
$$

This is the eta-reachability condition and implies that $\mathrm{e}_{y, \mathrm{i}}$ will converge to zero in finite time. When every component of 
$\mathrm{e}_{\mathcal{y}}(\mathrm{t})$ has converged to zero, a sliding motion takes place on the surface $\mathrm{S}$.

Remark: Note that this is not a global result. For any given $\rho$, there will be an initial condition of the observer (typically representing the very poor estimation of the initial conditions of the plant) so that (16) is not satisfied.

During sliding mode $\mathrm{e}_{y}(\mathrm{t})=\dot{\mathrm{e}}_{y}(\mathrm{t})=0$, and the error system defined by (11) and (12) can be written in a collapsed form as:

$$
\begin{gathered}
\dot{\mathrm{e}}_{1}(\mathrm{t})=\left(\mathrm{A}_{11} \mathrm{e}_{1}(\mathrm{t})+\mathrm{Lv}_{\mathrm{eq}}\right) \\
0=\left(\mathrm{A}_{21} \mathrm{e}_{1}(\mathrm{t})+\mathrm{v}_{\mathrm{eq}}\right)
\end{gathered}
$$

where $\mathrm{v}_{\mathrm{eq}}$ is the so-called equivalent output error injection that is required to maintain the sliding motion. This is the natural analogue of the equivalent control. Substituting $\mathrm{v}_{\mathrm{eq}}$ from (18) and (19) yields the following expression for the reduced-order sliding motion:

$$
\dot{\mathrm{e}}_{1}(\mathrm{t})=\left(\mathrm{A}_{11}+\mathrm{LA}_{21}\right) \mathrm{e}_{1}(\mathrm{t})
$$

This represents the reduced-order motion of order $n-p$ that governs the sliding mode dynamics. It can be shown that if $(A, C)$ is observable then $\left(A_{11} A_{21}\right)$ is also observable, and a matrix $L$ can always be chosen to ensure that the reduced-order motion in (20) is stable.

\section{B. Communication Rate and Threshold Design}

The average sensor communication rate is defined as:

$$
\gamma=\lim \sup _{\mathrm{T} \rightarrow+\infty}\left(\frac{1}{\mathrm{~T}+1}\right) \int_{\mathrm{t}=0}^{\mathrm{T}} \gamma_{\mathrm{t}} \mathrm{dt}
$$

The average rate $\gamma$ gives a general description of the network utilization. Below we consider the choice of the threshold $\zeta$ issue, given in (3), in order to achieve a desirable trade-off between communication rate and estimation quality.

\section{Communication Design Optimization}

In this section, the tradeoff between the estimation quality and the network bandwidth use as a constrained optimization problem is described. For this aim, the following cost functions $f_{1}$ and $f_{2}$ are defined:

$$
f_{1}(\zeta)=\gamma
$$

This function is related to number of the transmitted sensor measurement packets.

$$
f_{2}(\zeta)=\lim _{\mathrm{T} \rightarrow+\infty} \sqrt{\frac{1}{\mathrm{~T}+1} \int_{\mathrm{t}=0}^{\mathrm{T}} \mathrm{e}_{\mathrm{y}}(\mathrm{t})^{2} \mathrm{dt}}
$$

where $f_{2}$ is an estimation error RMS performance index.

Finally, both functions can be used to formulate a general performance index as following:

$$
f(\zeta)=\mathrm{Q} f_{1}(\zeta)+\mathrm{R} f_{2}(\zeta)
$$

where, $\mathrm{Q}$ and $\mathrm{R}$ are weighting matrices used to penalize the number of transmitted packets and the estimation quality respectively. Thus the co-design problem can be formulated as follows:

$$
\zeta^{*}=\operatorname{argminf}(\zeta)
$$

\section{SimUlation RESUlTS}

In this section, the effectiveness of the proposed co-design approach is illustrated with a numerical example. For simulation purposes, let us consider a nominal linear system described by (1) and modeled by:

$$
A=\left[\begin{array}{cc}
0 & 1 \\
-2 & 0
\end{array}\right], B=\left[\begin{array}{l}
0 \\
1
\end{array}\right], C=\left[\begin{array}{ll}
1 & 1
\end{array}\right]
$$

which represents a simple harmonic oscillator. For simplicity, we assume $\mathrm{u}(\mathrm{t})=0, \mathrm{D}=0$, and $\rho=1, \mathrm{Q}=5, \mathrm{R}=1$. The system's initial state is $\mathrm{x}_{0}=[0.5 ;-0.8]^{\mathrm{T}}$, and the observer has zero initial condition. Figure 2 shows the simulation diagram using Matlab/Simulink version R15.a. using a variable step ode45, Dormand Prince solver with a processor Intel(R) Core(TM) i5-8250U CPU.

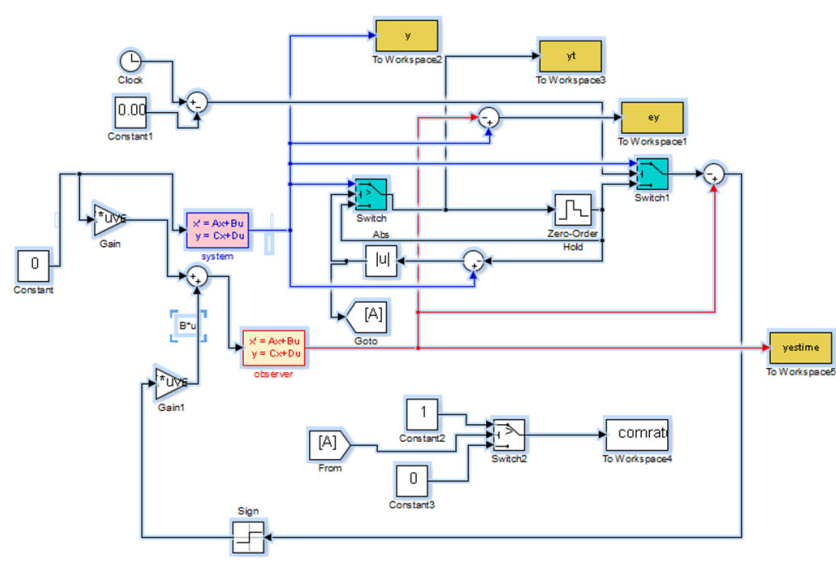

Fig. 2. Simulation diagram

In Table I the simulation results for different arbitrary values of $\zeta$ are summarized along with the optimal one for giving priority to the minimisation of energy consumption.

TABLE I. OPTIMIZATION OF ENERGY CONSUMPTION

\begin{tabular}{|c|c|c|c|c|}
\hline \multirow{2}{*}{ Case no } & \multicolumn{4}{|c|}{ Different values of $\boldsymbol{\zeta}$ with priority to energy minimization } \\
\cline { 2 - 5 } & $\boldsymbol{\zeta}$ & $\mathbf{r m s}(\mathbf{e y})$ & $\begin{array}{c}\text { Number of } \\
\text { transmissions }\end{array}$ & $\begin{array}{c}\text { Objective } \\
\text { function }\end{array}$ \\
\hline $\mathbf{1}$ & 0.1 & 0.0801 & 115 & 0.0806 \\
\hline $\mathbf{2}$ & 0.50 & 0.1059 & 22 & 0.1060 \\
\hline $\mathbf{3}$ & 1.50 & 0.3139 & 3 & 0.3139 \\
\hline Optimal & 0.9409 & 0.2291 & 9 & 0.2586 \\
\hline
\end{tabular}

Figures 3-6 show the system output $y_{t}$, the transmitted output $\mathrm{y}_{\mathcal{T}_{\mathrm{t}}}$ and the output estimation $\mathrm{y}_{\text {estim }}$ for different values of ऊ. Figure 6 is the optimal case. Excellent tracking of the output occurs after approximately $1.3 \mathrm{~s}$ and the output estimation error $\mathrm{e}_{\mathrm{y}}$ after approximately $1.0 \mathrm{~s}$ becomes zero, and the transmitted outputs are minimized. This is indicative of a high estimation quality with minimization of energy consumption. If we give priority to estimation rather than energy minimization, then $Q=1, R=5$. Table II exhibits the results of simulation for different values of $\zeta$. 


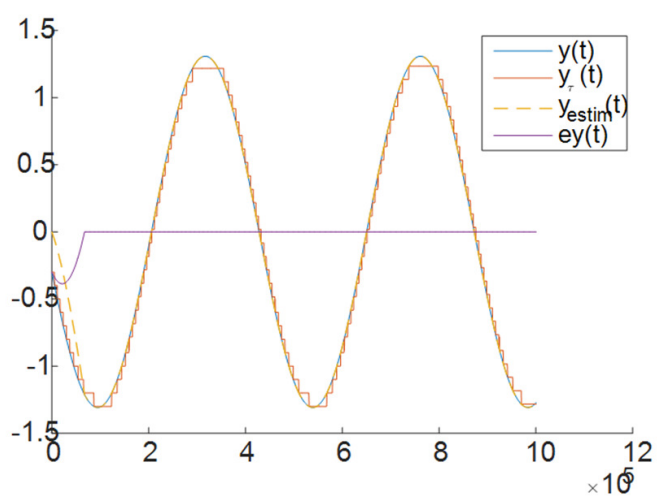

Fig. 3. $1^{\text {st }}$ case: The output $y_{t}$, the transmitted output $y_{\mathcal{T}_{t}}$, the output estimation error $\mathrm{e}_{\mathrm{y}}$, and the output estimation $\mathrm{y}_{\mathrm{estim}}$

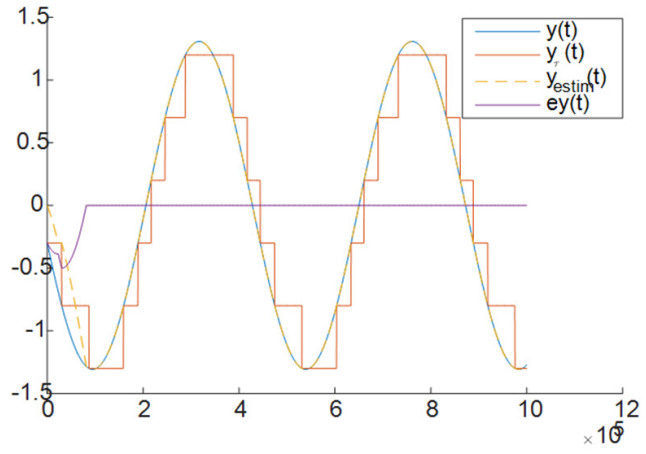

Fig. 4. $2^{\text {nd }}$ case: The output $\mathrm{y}_{\mathrm{t}}$, the transmitted output $\mathrm{y}_{\mathcal{T}_{\mathrm{t}}}$, the output estimation error $\mathrm{e}_{\mathrm{y}}$, and the output estimation $\mathrm{y}_{\text {estim }}$

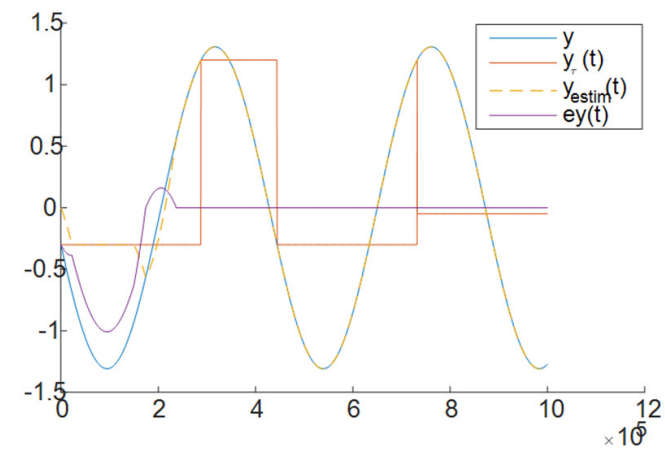

Fig. 5. $\quad 3^{\text {rd }}$ case: The output $\mathrm{y}_{\mathrm{t}}$, the transmitted output $\mathrm{y}_{\mathcal{T}_{\mathrm{t}}}$, the output estimation error $\mathrm{e}_{\mathrm{y}}$, and the output estimation $\mathrm{y}_{\text {estim }}$

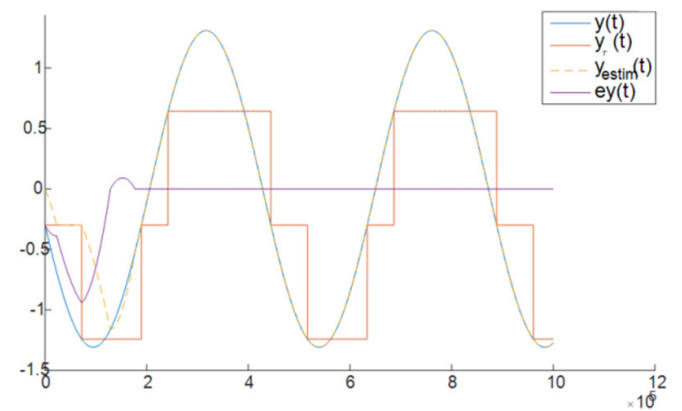

Fig. 6. Optimal case: The output $\mathrm{y}_{\mathrm{t}}$, the transmitted output $\mathrm{y}_{\mathcal{T}_{\mathrm{t}}}$, the output estimation error $\mathrm{e}_{\mathrm{y}}$, and the output estimation $\mathrm{y}_{\text {estim }}$
TABLE II. OPTIMIZATION OF ESTIMATION QUALITY

\begin{tabular}{|c|c|l|l|l|}
\hline \multirow{2}{*}{ Case no } & \multicolumn{4}{|c|}{ Different values of $\boldsymbol{\zeta}$ with priority to estimation } \\
\cline { 2 - 5 } & $\boldsymbol{\zeta}$ & $\mathbf{r m s}(\mathbf{e y})$ & $\begin{array}{c}\text { Number of } \\
\text { transmissions }\end{array}$ & $\begin{array}{c}\text { Objective } \\
\text { function }\end{array}$ \\
\hline $\mathbf{1}$ & 0.20 & 0.0801 & 57 & 0.4004 \\
\hline $\mathbf{2}$ & 1.00 & 0.2585 & 10 & 1.2926 \\
\hline $\mathbf{3}$ & 1.50 & 0.3139 & 3 & 1.5693 \\
\hline Optimal & 0.70 & 0.1564 & 14 & 0.7821 \\
\hline
\end{tabular}

Figures 7-10 plot the system output $\mathrm{y}_{\mathrm{t}}$, the transmitted output $\mathrm{y}_{\mathcal{T}_{\mathfrak{t}}}$, and the output estimation $\mathrm{y}_{\text {estim }}$ for different values of $\zeta$. Figure 10 shows the optimal case which clearly demonstrates a high estimation quality always with minimization of the transmitted output $\mathrm{y}_{\mathcal{J}_{\mathrm{t}}}$.

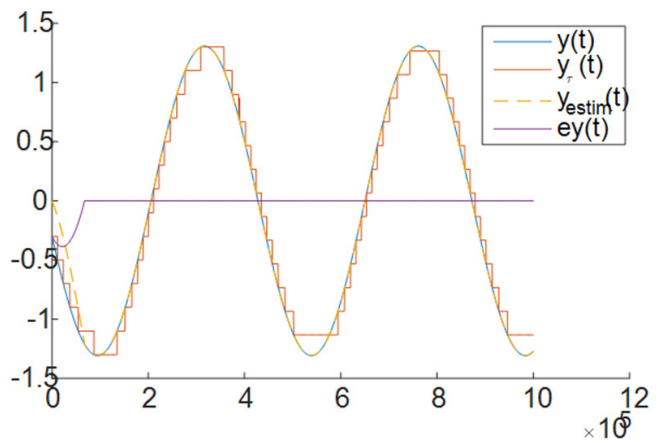

Fig. 7. $1^{\text {st }}$ case: The output $y_{t}$, the transmitted output $y_{\mathcal{T}_{t}}$, the output estimation error $\mathrm{e}_{\mathrm{y}}$, and the output estimation $\mathrm{y}_{\text {estim }}$

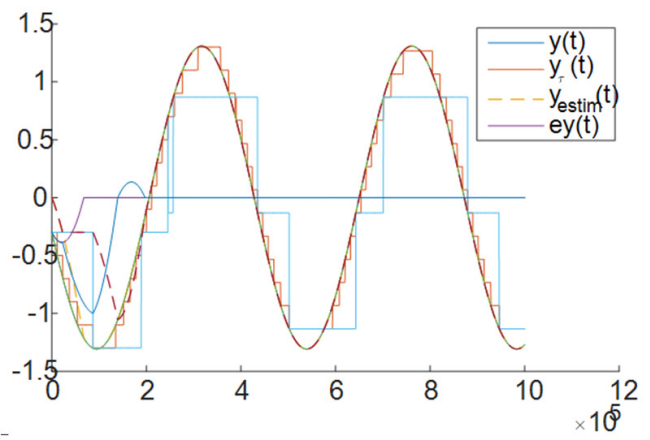

Fig. 8. $2^{\text {nd }}$ case: The output $\mathrm{y}_{\mathrm{t}}$, the transmitted output $\mathrm{y}_{\mathcal{J}_{\mathrm{t}}}$, the output estimation error $\mathrm{e}_{\mathrm{y}}$, and the output estimation $\mathrm{y}_{\mathrm{estim}}$

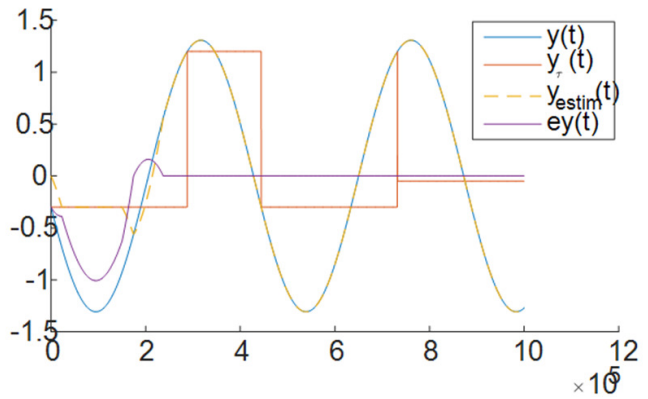

Fig. 9. $\quad 3^{\text {rd }}$ case: The output $y_{t}$, the transmitted output $y_{\mathcal{T}_{t}}$, the output estimation error $\mathrm{e}_{\mathrm{y}}$, and the output estimation $\mathrm{y}_{\mathrm{estim}}$ 


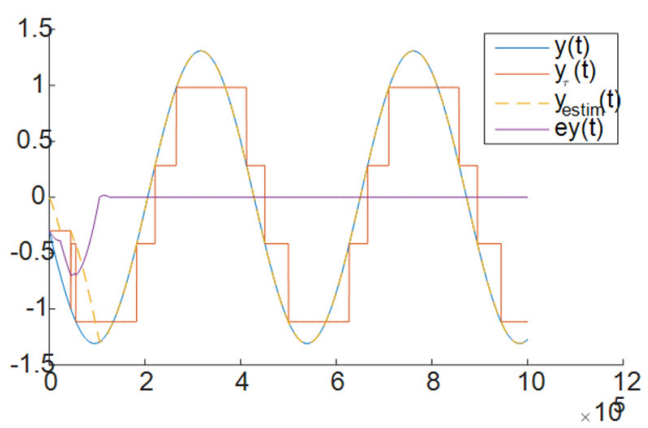

Fig. 10. Optimal case: The output $\mathrm{y}_{\mathrm{t}}$, the transmitted output $\mathrm{y}_{\mathcal{T}_{\mathrm{t}}}$, the output estimation error $\mathrm{e}_{\mathrm{y}}$, and the output estimation $\mathrm{y}_{\mathrm{estim}}$

\section{CONCLUSIONS}

Because of the verified benefits in terms of reduced energy consumption and better resource utilization compared to traditional designs, event-triggered estimation methods can be widely adopted in industrial applications. For instance, in achieving optimal state estimation over a shared network we use a sliding mode observer associated with an event triggering mechanism. It was shown that by the introduction of an event based technique and the communication design optimization with a corresponding estimator by adopting sliding mode, reduction of the estimation error and of the sensor transmission can be achieved. The simulation results illustrate the effectiveness of the proposed sliding mode observer in terms of decreased estimation error and reduced sensor transmitted information.

\section{REFERENCES}

[1] Y. Shtessel, C. Edwards, L. Fridman, A. Levant, Sliding mode control and observation, Birkhauser Basel, 2014

[2] S. Hui, S. H. Zak, "Observer design for systems with unknown inputs", International Journal of Applied Mathematics and Computer Science, Vol. 15, No. 4, pp. 431-446, 2005

[3] B. Walcott, S. H. Zak, "State observation of nonlinear uncertain dynamical systems", IEEE Transactions on Automatic Control, Vol. 32 No. 2, pp. 166-170, 1987

[4] B. W. Edwards, S. K. Spurgeon, R. J. Patton,"Sliding mode observers for fault detection and isolation", Automatica, Vol. 36, pp. 541-553, 2000

[5] O. Aydogdu, M. L. Levent, "Kalman state estimation and LQR assisted adaptive control of a variable loaded servo system", Engineering, Technology \& Applied Science Research, Vol. 9, No. 3, pp. 4125-4130, 2019

[6] S. V. Drakunov, "An adaptive quasi-optimal filter with discontinuous parameters", Automation and Remote Control, Vol. 44, No. 9, pp. $1167-$ 1175,1983

[7] S. V. Drakunov, "Sliding mode observers based on equivalent control method", 31 st IEEE Conference on Decision and Control, Tucson, USA, December 16-18, 1992

[8] S. V. Drakunov, V. I. Utkin, "Sliding mode observers. Tutorial", in: Proceedings of 34th IEEE Conference on Decision and Control, Vol. 4, pp. 3376-3378, IEEE, 1995

[9] V. I. Utkin, "Sliding modes in control and optimization", in: Communications and Control Engineering Series, Springer-Verlag, 1992

[10] J. Sijs, B. Noack, M. Lazar, U. D. Hanebeck, "Time-periodic state estimation with event-based measurement updates", in: Event-Based Control and Signal Processing. CRC Press, 2016

[11] D. Shi, L. Shi, T. Chen, Event-based state estimation, Springer, 2016
[12] S. P. Singh, S. C. Sharma, "A novel energy efficient clustering algorithm for wireless sensor networks", Engineering, Technology \& Applied Science Research, Vol. 7, No. 4, pp. 1775-1780, 2017

[13] L. Li, M. Lemmon, X. Wang, "Event-triggered state estimation in vector linear processes", 2010 American Control Conference, Baltimore, USA, June 30-July 2, 2016

[14] R. Cogill, S. Lall, J. P. Hespanha, "A constant factor approximation algorithm for event-based sampling", in: Perspectives in Mathematical System Theory, Control, and Signal Processing, Springer, 2010

[15] S. Trimpe, "Predictive and sell triggering for event-based state estimation", IEEE 55th Conference on Decision and Control, Las Vegas, USA, December 12-14, 2016 